\title{
EchoGéo
}

30 | 2014

Varia

\section{Intersectionnalité, migrations et travail domestique : lectures croisées en France et aux États-Unis}

\section{Sophie Blanchard}

\section{OpenEdition}

Journals

Electronic version

URL: https://journals.openedition.org/echogeo/14073

DOI: 10.4000/echogeo.14073

ISSN: 1963-1197

\section{Publisher}

Pôle de recherche pour l'organisation et la diffusion de l'information géographique (CNRS UMR 8586)

Electronic reference

Sophie Blanchard, "Intersectionnalité, migrations et travail domestique : lectures croisées en France et aux États-Unis", EchoGéo [Online], 30 | 2014, Online since 16 January 2015, connection on 31 July 2021. URL: http://journals.openedition.org/echogeo/14073; DOI: https://doi.org/10.4000/echogeo. 14073

This text was automatically generated on 31 July 2021.

EchoGéo est mis à disposition selon les termes de la licence Creative Commons Attribution - Pas d'Utilisation Commerciale - Pas de Modification 4.0 International (CC BY-NC-ND) 


\title{
Intersectionnalité, migrations et travail domestique : lectures croisées en France et aux États-Unis
}

\author{
Sophie Blanchard
}

1 La notion d'intersectionnalité émerge dans les années quatre-vingt-dix pour rendre compte de l'imbrication des discriminations sexistes et racistes subies par les femmes afro-américaines et dénoncées depuis les années soixante-dix par le black feminism, à l'intersection de rapports sociaux de sexe, de classe et de race $^{1}$ (Crenshaw, 1991). Formulée par la juriste K. Crenshaw, la notion a été rapidement adoptée dans les milieux académiques et militants anglophones: l'intersectionnalité devient un paradigme utilisé pour comprendre l'articulation d'inégalités et de discriminations multiples (Bilge, 2009). Dans la bibliographie scientifique française, on trouve des approches comparables qui ne reprennent souvent pas le terme d'intersectionnalité : D. Kergoat, envisageant les rapports sociaux comme des rapports de production matérielle et idéelle entre deux groupes, utilise le terme de «consubstantialité des rapports sociaux» (Kergoat, 2009), C. Hamel lui préfère celui d'«intrication des rapports sociaux de sexe, de race, d'âge et de classe » (Hamel, 2003).

2 Les approches croisant genre, race et classe sont particulièrement utiles pour étudier les liens entre migrations, minorités et travail domestique. En effet, l'étude du travail domestique invite à croiser trois dimensions. Le sexe tout d'abord, puisque les travailleur-ses domestiques sont en grande majorité des femmes (Rollins, 1985). La race et le statut de migrant ensuite : les emplois domestiques sont en grande partie pourvus par des femmes migrantes ou issues de minorités, amérindiennes en Amérique latine, afro-américaines aux États-Unis, Philippines en Asie (Mozère, 2005). La classe enfin, car le travail domestique renvoie à un métier de service dévalorisé et subordonné. Le travail domestique, qui émerge comme objet d'étude en géographie française, a constitué un prisme pour étudier les femmes migrantes ou des femmes racialisées et altérisées en position de minorité. Ces études qui mettent l'accent sur les rapports de domination interrogent surtout des espaces éloignés: l'Afrique du Sud (Bénit et 
Morange 2004), la Bolivie (Blanchard, 2014), le Liban (Cattan, 2012 ; Dahdah, 2014), ou encore l'Inde (Varrel, 2002). Le thème a fait l'objet d'approches sociologiques et anthropologiques plus anciennes et plus diversifiées. Des numéros spéciaux de revues ont été consacrés au travail domestique : un numéro de la Revue Tiers-Monde en 2002 (Destremeau et Lautier, 2002) sur les femmes en domesticité, et un numéro de la revue Travail, genre et sociétés de 2009 portant sur les "Domestiques d'ici et d'ailleurs" (Drouilleau, 2009). Ces études du travail domestique mettent elles aussi souvent l'accent sur les domestiques au Sud, mais des travaux sur le contexte français inscrits dans la sociologie du travail se développent (Angeloff, 2003 ; Avril, 2014).

L'analyse des travailleuses domestiques et des conditions d'exercice des emplois domestiques à travers une approche intersectionnelle croisant genre, race et classe semble donc particulièrement riche. À travers les lectures croisées de deux textes dont les contextes de production sont très différents, l'objectif est ici de mettre en lumière la façon dont la notion d'intersectionnalité permet de prendre en compte les parcours des travailleuses domestiques migrantes. Le premier texte, De la servitude au travail de service: les continuités historiques de la division raciale du travail reproductif payé, est un essai d'Evelyn Nakano Glenn portant sur la division raciale du travail reproductif payé aux États-Unis (Glenn, 2009). Sociologue, Evelyn Nakano Glenn est une des pionnières des études de l'emploi domestique croisant le genre et la race (Glenn, 1986); elle a ensuite poursuivi cette étude pendant plusieurs décennies (Glenn, 2012). Ce texte, publié pour la première fois en 1992, s'inscrit dans les women studies, champ de recherches florissant aux États-Unis depuis les années quatre-vingt (Ehrenreich et Hoschchild, 2003; Rollins, 1985). L'ouvrage de Christelle Avril, Les Aides à domicile. Un autre monde populaire, rend compte de travaux plus récents : publié en 2014, ce livre est issu d'une thèse de sociologie soutenue en 2007. Il s'inscrit dans une sociologie du travail « ouverte et décloisonnée » fondée sur des approches ethnographiques (Avril et al., 2010). Ces deux approches sociologiques très différentes par leurs objets et leurs démarches montrent des parallèles entre la construction racialisée de l'emploi domestique aux États-Unis au cours du XX ${ }^{e}$ siècle et les relations interraciales existant au sein d'un groupe d'aides à domicile en France au début du XXI ${ }^{\mathrm{e}}$ siècle.

\section{Aux États-Unis, des constructions genrées et racialisées de l'emploi domestique}

Dans ce texte de synthèse publié en 1992 et traduit ici en français, Evelyn Nakano Glenn reprend certains de ses travaux antérieurs sur les femmes japonaises dans l'emploi domestique (Glenn, 1986) et les croise avec de nombreuses études d'autres auteurs pour analyser la construction genrée et racisée du travail domestique/reproductif aux ÉtatsUnis (Glenn, 2009). Pendant la première moitié du XX siècle, des femmes « racialiséesethnicisées $»^{2}$ étaient employées comme domestiques au sein des foyers blancs : Afroaméricaines dans le Sud, Mexicaines et Chicanas dans le Sud-Ouest, Japonaises dans l'Ouest (nord de la Californie, Hawaï). Ces constructions évoluent: par exemple en Californie, les domestiques furent d'abord des hommes, domestiques et cuisiniers chinois à San Francisco à la fin du XIX ${ }^{e}$ siècle, domestiques japonais à Honolulu au début du XXe siècle, puis à partir de 1907, débutèrent des restrictions sur l'immigration de travail des hommes japonais. Commença alors une émigration significative de femmes japonaises vers la Californie ou Hawaï. Dans les années 1930, environ la moitié 
des femmes américaines d'origine japonaise (de $1^{\text {ère }}$ ou de $2^{\mathrm{e}}$ génération), dans la région de San Francisco et à Honolulu, étaient employées comme domestiques. Pendant la Seconde guerre mondiale, les Japonais se retrouvèrent dans des camps d'internement. Après la guerre, pour sortir des camps, ils devaient justifier d'un emploi et d'un domicile. Beaucoup de femmes, orientées par des agences de recrutement de domestiques, ont alors accepté des emplois de servantes à domicile : ayant perdu leurs maisons et leurs biens, elles n'avaient guère de choix. Des stéréotypes ethniques ont été construits à propos de ces différents groupes de femmes travailleuses domestiques, considérées par leurs employeurs comme particulièrement «aptes" au travail domestique. Les femmes japonaises par exemple étaient réputées être calmes et frugales : on retrouve une évocation de ces stéréotypes ${ }^{3}$ dans un récent roman de Julie Otsuka retraçant l'histoire des migrantes japonaises aux États-Unis (Otsuka, 2013). Les autres secteurs d'emploi leur étaient relativement fermés, hormis l'agriculture et les emplois de vendeuses dans des magasins d'art oriental (Glenn, 2009, p. 38).

Evelyn Nakano Glenn montre ainsi de quelle façon la division sexuelle et raciale du travail est liée à des histoires migratoires. La race et le genre sont des systèmes socialement construits, qui voient le jour dans des circonstances particulières et sont susceptibles d'évoluer lorsque ces circonstances changent (Glenn, 2009, p. 56). Pour les migrants japonais, les circonstances ont changé, il y a longtemps qu'on ne parle plus de "péril jaune ${ }^{4}$, et que l'immigration de travailleurs japonais pauvres et non qualifiés aux États-Unis a cessé. Le stéréotype a donc changé. Au-delà du cas des femmes japonaises migrantes, l'analyse vaut pour l'ensemble des «femmes américaines d'origine africaine, mexicaine et japonaise (qui) ont été poussées vers le travail domestique par une combinaison de besoins économiques, d'opportunités limitées et de mécanismes d'orientation éducationnels et professionnels" (Glenn, 2009, p. 56). Leur association avec le travail domestique était ensuite utilisée pour les placer dans une position d'infériorité. On retrouve des associations/assignations similaires aujourd'hui dans le secteur du travail domestique en France, par exemple dans les stéréotypes qui se sont forgés à propos des gardes d'enfants originaires d'Afrique subsaharienne (Ibos, 2012). Cela renforce l'idée que la relation entre la race, le genre et la classe crée des catégories positionnées qui ne prennent sens que dans la relation qui les lie. Ces catégories évoluent et les relations entre la race, le genre et la classe se recomposent aussi au gré des évolutions du marché de l'emploi. Aujourd'hui, les femmes afro-américaines et latinas sont nettement surreprésentées dans les emplois de femme de ménage, les services de nettoyage et d'entretien, les emplois de plongeuse, d'aide-cuisinière, ou d'aide-soignante, soit des emplois "sales et invisibles " (Glenn, 2009, p. 43). On observe des hiérarchies de race et de genre dans le travail hospitalier par exemple. En retraçant la façon dont la race et le genre ont été formés dans une sphère du travail des femmes, Evelyn Nakano Glenn montre qu'il s'agit « de systèmes socialement construits faits de rapports - incluant des symboles, des croyances normatives et des pratiques organisés autour de différences perçues» (Glenn, 2009, p. 56). Le texte ici présenté fait référence au "travail reproductif payé », et préfigure les analyses du travail du care qu'Evelyn Nakano Glenn a menées par la suite dans un ouvrage de référence : Forced to Care, Coercion and Caregiving in America (Glenn, 2012). La vision critique du care qu'elle développe rappelle à quel point cette notion ambivalente, qui s'est imposée à la fois dans le champ de la recherche et dans le champ politique, se trouve au croisement des rapports sociaux de classe, de sexe et de race et repose sur 
des formes de racisation du travail (Kergoat, 2012). Des parallèles peuvent être établis avec le travail des aides à domicile étudié par Christelle Avril.

\section{En France, les relations interraciales dans le monde des aides à domicile}

6 Les discriminations croisées liées à l'origine sociale et ethnique jouent en effet un rôle dans la façon dont les aides à domicile exercent et perçoivent leur travail ; elles influent également sur les attentes des employeurs et des responsables des associations. L'ouvrage de Christelle Avril (2014), issu d'une thèse de sociologie soutenue en 2007, se fonde sur des entretiens et des observations participantes avec 75 aides à domicile dans le cadre d'une association située dans une commune de banlieue parisienne ${ }^{5}$. Environ la moitié des femmes enquêtées sont immigrées, filles d'immigrées (d'Afrique du Nord ou d'Afrique subsaharienne) ou originaires des DOM/TOM. À l'échelle nationale, Christelle Avril établit que la part des étrangères, des immigrées et des filles d'immigrées est plus élevée chez les aides à domicile que dans l'ensemble des femmes en emploi. Cette surreprésentation est nettement plus marquée dans l'agglomération parisienne où a eu lieu l'enquête : une aide à domicile sur deux y est née étrangère.

7 L'enquête de terrain menée par Chistelle Avril fait apparaître l'importance des relations interraciales entre les aides à domicile, dans une association dont l'ambiance est qualifiée de «souvent raciste». Elle pose donc «la question de savoir ce que ce nouveau type de relations, soulevant le problème de l'imbrication de la classe, du genre et de la « race », implique pour la recomposition interne des milieux populaires et pour les liens des milieux populaires avec le reste de l'espace social» (Avril, 2014, p. 24). Les relations interraciales décrites créent des lignes de clivage entre aides à domicile, les aides à domicile «noires» ou " arabes» (termes employés par les femmes enquêtées alors que celui de «blanches » n'est jamais employé) étant tenues à distance par une partie de leurs collègues.

8 Ces relations racialisées, qui n'ont pas toujours de connotation explicitement infériorisante, participent d'une « ambiance raciste » qui voit se constituer un « Nous » (les «autochtones») positionné face à «Elles» (les « Noires» et les «Arabes»). De telles ambiances racistes ont été décrites dans d'autres milieux professionnels, par exemple dans le secteur du bâtiment où l'identification ethnique des travailleurs, sous forme de plaisanteries qui jouent sur les frontières ethniques, participe d'un processus d'ethnicisation (Jounin, 2004). Parmi les stéréotypes évoqués dans l'ouvrage de Christelle Avril, l'idée que les femmes arabes seraient des "victimes", dominées et exploitées par leurs maris, qu'elles travailleraient à l'excès, qu'elles "courent partout ", alors que les femmes noires seraient «nonchalantes » aux yeux de leurs collègues et auraient une activité sexuelle débridée (Avril, 2014, p. 180). On retrouve là des stéréotypes participant de la racialisation des féminités, à travers l'assignation des femmes noires et arabes à des rôles sexuels érotisés (Guénif, 2005), stéréotypes mis en évidence par Karima Ramdani dans les clips de raps donnant à voir des représentations érotisés des corps des femmes noires (Ramdani, 2011). Ce racisme est partagé par une partie des employeurs : certains précisent explicitement qu'ils ne veulent pas d'aide à domicile noire ou arabe, et leurs demandes sont toujours satisfaites. Le fait de s'occuper de personnes âgées sert alors à légitimer la discrimination raciale, les demandes des 
personnes âgées étant acceptées sans commentaires au prétexte de leur âge et de leur appartenance générationnelle.

Les deux groupes, «Nous » et "Elles », n'ont de surcroît pas la même conception de l'aide à domicile. Les relations racialisées décrites au sein de l'association étudiée produisent des formes différenciées de rapport au travail et de gestion de la main d'œuvre, ce que l'on observe aussi dans d'autres travaux consacrés au rapport au travail, qui est sexué mais dépend aussi de la classe et de la race (Kergoat, 2012), et donne parfois lieu à une forme de gestion raciste des travailleurs (Jounin, 2004). Les femmes «autochtones" se conçoivent plus souvent comme des professionnelles du ménage, elles ont des horaires maîtrisés, effectuent des tâches précisément définies, et développent peu de relations personnelles avec les personne âgées sauf de manière ponctuelle en fonction des affinités. Les femmes "racialisées" se conçoivent plus souvent comme des professionnelles de la vieillesse : elles ont fait plus d'études et sont souvent déclassées professionnellement, ce qui rappelle d'autres travaux sur les trajectoires professionnelles de femmes noires diplômées (Diop, 2011). Elles entretiennent des relations plus personnelles avec les personnes âgées auprès desquelles elles sont plus fortement impliquées: elles les font manger, font leur toilette. Ces femmes acceptent de faire plus d'interventions, notamment le soir et le week-end, en partie parce qu'elles sont économiquement plus vulnérables car elles sont plus souvent seules avec des charges de famille, et qu'elles ne disposent pas du réseau de ressources locales des femmes autochtones. Elles ont aussi souvent fait préalablement l'expérience du travail domestique à demeure, en travaillant comme "garde-malade 24 heures sur 24 ", et celle de la discrimination raciale. Leur période d'essai est plus longue et plus rude: les responsables de l'association leur confient d'emblée des cas plus lourds, et ne leur donnent que très peu d'heures par semaine au début. Elles seraient aussi plus surveillées et plus facilement licenciées. Les responsables de l'association et les employeurs n'ont pas les mêmes attentes envers ces femmes, ils ne portent pas le même regard sur leur travail et sur la façon dont elles doivent le faire. Les formes de catégorisation fondées sur le sexe et la race débordent donc du cadre des relations interraciales entre les aides à domicile.

\section{Conclusion}

10 Il peut paraitre paradoxal de réfléchir à la notion d'intersectionnalité à travers deux textes qui n'emploient pas le terme, tout en analysant les relations entre genre, race et classe. La lecture croisée de ces deux textes laisse cependant entrevoir ce que la démarche intersectionnelle peut apporter à l'étude de groupes sociaux localisés et racialisés. Cette démarche est donc riche de promesses pour une géographique sociale des migrations. 


\section{BIBLIOGRAPHY}

Angeloff T., 2003. Employées de maison, aides à domicile : un secteur paradoxal. In Weber F. et al., Charges de famille. Dépendance et parenté dans la France contemporaine. La Découverte, p. 165-186.

Avril C., Cartier M., Serre D., 2010. Enquêter sur le travail. Concepts, méthodes, récits. Paris, La Découverte, $283 \mathrm{p}$.

Avril C., 2014. Les aides à domicile. Un autre monde populaire. Paris, La Dispute, Paris, 289 p.

Bénit C., Morange M., 2004. Les domestiques, la ville et l'accès à l'emploi au Cap et à Johannesburg : logiques de proximité et logiques de réseau. Revue Tiers Monde, n 179, p. 539-565. Bilge S., 2009. Théorisations féministes de l'intersectionnalité. Diogène, 1/2009 (n²25), p. 70-88. Blanchard S., 2014. Migration féminine et "condition domestique". De l'apprentissage à la professionnalisation, trajectoires de travailleuses domestiques andines à Santa Cruz. Revue TiersMonde, $\mathrm{n}^{\circ}$ 217, p. 147-162.

Cattan N., 2012. Trans-territoire. Repenser le lieu par les pratiques spatiales de populations en position de minorité. L'Information géographique, vol. 76, p. 57-71.

Crenshaw K. W., 1991. Mapping the Margins: Intersectionality, Identity Politics, and Violence against Women of Color. Stanford Law Review, vol. 43, n 6, p. 1241-1299.

Dahdah A., 2014. "Maid in Lebanon". Lebanon and the world domestic service market. In Anteby L., et al., (dir.), Borders, Migrations and mobilities. Perspectives from the Mediterranean. Peter Lang, Bruxelles, p. 131-148.

Destremeau B., Lautier B., 2002. Introduction : Femmes en domesticité. Les domestiques du Sud, au Nord et au Sud. Revue Tiers Monde, $\mathrm{n}^{\circ}$ 170, p. 249-264.

Diop C., 2011. Les femmes noires diplômées face au poids des représentations et des discriminations en France. Hommes \& Migrations, 4/2011 ( $n^{\circ}$ 1292), p. 92-102.

Drouilleau F. et al., 2009. Domestiques d'ici et d'ailleurs. Travail, genre et sociétés, n²2, p. 25-30. Ehrenreich B., Hoschchild A. R., 2003. Global Woman. Nannies, Maids and Sex Workers in the New Economy. New York, Metropolitan Books, 328 p.

Fassi, D., Fassin E., 2009. De la question sociale à la question raciale ? Représenter la société française. Paris, La Découverte, p 238-256.

Glenn E.N., 1986. Issei, Nisei, Warbride: Three Generations of Japanese American Women in Domestic Service. Philadelphie, Temple University Press, 290 p.

Glenn E.N., 2009. De la servitude au travail de service : les continuités historiques de la division raciale du travail reproductif payé. In Dorlin E. (dir.), Sexe, race, classe. Pour une épistémologie de la domination. Paris, PUF, p. 21-63.

Glenn E.N., 2012. Forced to Care, Coercion and Caregiving in America. Cambridge, Harvard University Press, $272 \mathrm{p}$.

Guénif-Souilamas N., 2005. La réduction à son corps de l'indigène de la République. In La fracture coloniale. Paris, La Découverte, p. 199-208. 
Hamel C., 2003. L'intrication des rapports sociaux de sexe, de race, d'âge et de classe : ses effets sur la gestion des risques d'infection par le VIH chez les français descendant de migrants du Maghreb. Thèse de doctorat en Ethnologie et anthropologie sociale, dir. M.-E. Handman, Paris, EHESS, 1440 p.

Ibos C., 2012. Qui gardera nos enfants? Les nounous et les mères. Paris, Flammarion, 288 p.

Jounin N., 2004. L'ethnicisation en chantiers. Reconstructions des statuts par l'ethnique en milieu de travail. Revue européenne des migrations internationales, vol. 20, n 3-2004, p. 103-126.

Kergoat D., 2009. Dynamique et consubstantialité des rapports sociaux. In Dorlin E. (dir.), Sexe, race, classe. Pour une épistémologie de la domination. Paris, PUF, p. 125-140.

Kergoat D., 2012. Se battre, disent-elles... Paris, La Dispute, 355 p.

Mozère L., 2005. Les domestiques philippines à Paris. Migrations Société, Paris, vol. 17, nº 99-100, Femmes dans la migration, 2006, p. 217-228.

Otsuka J, 2013. Certaines n'avaient jamais vu la mer. Paris, Pocket, $143 \mathrm{p}$.

Ramdani K., 2011. Bitch et Beurette, quand féminité rime avec liberté. Volume !, 2/ 2011 (8:2), p. 13-39.

Rollins J., 1985. Between Women. Domestics and their Employers. Philadelphie, Temple University Press, $256 \mathrm{p}$.

Varrel A., 2002. Itinéraires du travail domestique en Inde : les filles d'Erayiur. Revue Tiers-Monde, vol. 43, n 170, p. 353-371.

\section{NOTES}

1. Le terme de race renvoie ici à un construit social permettant de rendre compte des discriminations et des catégorisations (Fassin et Fassin, 2009).

2. E.N. Glenn utilise l'expression de «femmes racialisées-ethnicisées » pour désigner «des groupes socialement construits comme racialement et culturellement différents des Américains d'origine européenne » (Glenn, 2009, p. 23), mais parle d'une construction racisée et genrée du travail. D'autres travaux préfèrent le terme de racisation (Kergoat, 2012) ou de relations interraciales (Avril, 2014) pour évoquer les constructions fondées sur l'origine ethno-raciale. La multiplicité des termes employés renvoie à la difficulté de nommer ces constructions tout en se démarquant d'une vision essentialiste de la race (Fassin et Fassin, 2009).

3. « Nous ne parlions guère. Mangions peu. Nous étions douces. Nous étions bonnes. Nous ne causions jamais de problèmes et les laissions faire de nous ce qu'elles voulaient » (Otsuka, 2013, p. 143).

4. Terme employé aux Etats-Unis à l'encontre des Japonais au début du XX $\mathrm{XX}^{\mathrm{e}}$ siècle.

5. Cette commune est anonymisée. 


\section{ABSTRACTS}

This paper confronts two studies of women working in domestic service: an essay by Evelyn Nakano Glenn about the racialized construction of domestic work in the United Stades during the XXth century and an ethnographic study of domestic helpers in XXIth century France by Christelle Avril. These studies illustrate how intersectionnality can be a valuable tool to analyze the condition of racialized domestic workers.

À travers une lecture croisée de deux textes, un essai d'Evelyn Nakano Glenn sur la construction racialisée de l'emploi domestique aux États-Unis au cours du $\mathrm{XX}^{\mathrm{e}}$ siècle et un ouvrage de Christelle Avril sur le travail des aides à domicile en France au début du XXI ${ }^{\mathrm{e}}$ siècle, les réflexions qui suivent soulignent l'intérêt de la notion d'intersectionnalité pour étudier la condition des travailleuses domestiques migrantes et/ou racialisées.

\section{INDEX}

Mots-clés: ethnicité, genre, intersectionnalité, migration, travail domestique

Keywords: ethnicity, gender, intersectionnality, migration, domestic work

\section{AUTHOR}

\section{SOPHIE BLANCHARD}

Sophie Blanchard, sophie.blanchard@u-pec.fr est géographe à l’Université Paris-Est Créteil.Elle a publié récemment :

- Blanchard S., 2014. Migration féminine et « condition domestique » : de l'apprentissage à la professionnalisation. Revue Tiers Monde, n² 217, p. 147-162.

- Blanchard S., 2014. Mobilités et pratiques de loisirs des étudiants débutants. Le cas de l'Université de Créteil. Espaces et sociétés, n 159, p. 127-146. 